\title{
Impact of electric vehicles charging demand on distribution transformers in an office area and determination of flexibility potential
}

\author{
M.A. van den Berg ${ }^{\text {a,* }}$, I. Lampropoulos ${ }^{\mathrm{b}}$, T.A. AlSkaif ${ }^{\mathrm{b}, \mathrm{c}}$ \\ ${ }^{a}$ Energy Markets \& Technology, DNV GL, Utrechtseweg 310, 6812 AR Arnhem, The Netherlands \\ ${ }^{\mathrm{b}}$ Copernicus Institute of Sustainable Development, Utrecht University, Princetonlaan 8a, 3584 CB Utrecht, The Netherlands \\ ${ }^{\mathrm{c}}$ Information Technology Group, Wageningen University and Research, Hollandseweg 1, 6706 KN Wageningen, The Netherlands
}

\section{A R T I C L E I N F O}

\section{Article history:}

Received 10 August 2020

Received in revised form 27 January 2021

Accepted 15 February 2021

Available online 26 February 2021

\section{Keywords:}

Electric vehicles

Charging power

Grid impact

Demand flexibility

\begin{abstract}
A B S T R A C T
Electric vehicles (EVs) have a high potential in reducing greenhouse gas emissions and are able to achieve other advantages such as a reduction in local air pollution and increasing energy security. As a result, EVs are rapidly increasing in popularity, electrifying the transportation sector. This poses a serious problem for the grid as existing distribution grids were mainly sized in the pre-EV era. In this paper, a method is proposed to determine the charging demand of future EV fleets in an office area and determine its flexibility potential. Office charging is studied as it differs from residential charging and a limited number of studies focused solely on office charging. The study is an empirical study and is based on analysing real transaction data of $42 \mathrm{EVs}$ charging for over a year at Utrecht Science Park, Utrecht, the Netherlands, the considered case study. The transaction data allows for an examination of the impacts of future EV charging demand in an office area. The results based on a future scenario study show that in 2050, 4 out of 7 studied transformers are overloaded. This is followed by an analysis on the mitigation of the determined impact. This analysis also determined the flexibility in EV demand, around 50\% of the EV demand can be delayed for more than $8 \mathrm{~h}$. When this flexibility is used, overloading of 3 out of 4 transformers could be mitigated. This paper shows that placement of charging stations should be strategically performed, considering the capacity of the network and taking into account the forecasted load due to EVs charging.
\end{abstract}

(c) 2021 Elsevier Ltd. All rights reserved.

\section{Introduction}

The $\mathrm{CO}_{2}$ emissions from the transportation sector is one of the biggest contributors to global warming and consequently, anthropogenic climate change [1]. In an effort to mitigate the adverse impacts of anthropogenic global warming and climate change, EVs are promoted worldwide. This is due to their potential to mitigate $\mathrm{CO}_{2}$ emissions when the electrical energy used to charge the $\mathrm{EV}$ is generated by a renewable energy source (RES). In addition, for local air quality EVs offer clear benefits, mainly due to zero tailpipe emissions at street level of pollutants such as $\mathrm{NO}_{\mathrm{x}}$ and particulate matter, reducing the local polluted emissions [2]. This is in particular important for densely populated urban areas [3].

In Europe, the Dutch government has established the ambition to have all passenger cars be zero-emission by 2050 [4], which equals to 9.5 million EVs in 2050 in the Netherlands. Not only the national government, but also local governments have shown interest in EVs. An example is the municipality of Utrecht, which

\footnotetext{
* Correspondence to: Copernicus Institute of Sustainable Development, Utrecht University, Princetonlaan 8a, 3584 CB Utrecht, The Netherlands.

E-mail address: marianvandenberg@outlook.com (M.A. van den Berg).
}

aims to be a climate neutral city in 2030 and achieve a $30 \%$ reduction in $\mathrm{CO}_{2}$ emissions by stimulating EVs [5]. The combination of these policy initiatives and advancements in battery and EV technology will likely result in an extensive use of EVs in the near future. The ambition of the Dutch government to stimulate the uptake of EVs should result in a passenger vehicle fleet which consists solely of EVs in 2050. However, it remains unclear how the charging infrastructure will develop in order to supply the EV charging demand [3].

EVs require the use of batteries with high energy density and with large electric demand requirements. These batteries must be charged frequently, often at the level of the low-voltage (LV) distribution grid. As a result, distribution grid transformers that were sized before EV integration may become overloaded and might be unreliable to support a large deployment of EVs. Prolonged overloading of a distribution grid transformer may lead to failure in supplying power. In this context, several studies have looked into the other impacts on parameters of the distribution network's system design and operation such as; high current demand [6], power flows [6], load unbalance [7], higher energy losses [8], voltage profile [9], harmonics [10] and peak load [11]. The combination of all these impacts might require to 


\begin{tabular}{|c|c|}
\hline \multicolumn{2}{|c|}{ Nomenclature } \\
\hline \multicolumn{2}{|l|}{ Indices } \\
\hline $\mathrm{q}$ & Index of transaction in dataset \\
\hline $\mathrm{j}$ & Index of EV in dataset \\
\hline \multicolumn{2}{|l|}{ Symbols } \\
\hline $\mathrm{t}_{\text {plug-in }}^{\mathrm{q}}$ & The plug-in time for transaction $q[-]$ \\
\hline $\mathrm{t}_{\text {plug-out }}^{\mathrm{q} \text { qug-in }}$ & The plug-out time for $\mathrm{t}$ transaction $q[-]$ \\
\hline $\mathrm{E}_{\mathrm{req}}^{\mathrm{q}}$ & The total charged energy during each transaction $q[\mathrm{kwh}]$ \\
\hline $\mathrm{H}_{\text {DUR,max }}$ & The maximum connection time of an EV $j$ that occurs in the dataset [h] \\
\hline $\mathrm{H}_{\text {plug-in,max }}$ & The maximum plug-in hour of an EV $j$ that occurs in the dataset [-] \\
\hline $\mathrm{N}_{\mathrm{EV}, 2050}$ & The estimated EV fleet in $2050[-]$ \\
\hline $\mathrm{H}_{\text {plug-in }}^{\mathrm{q}}$ & Plug-in hour of the day for transaction $q[-]$ \\
\hline $\mathrm{T}_{\text {connect }}^{\mathrm{q}}$ & The connection time for transaction $q[\mathrm{~h}]$ \\
\hline $\mathrm{P}_{\max }$ & Maximum power charged by the EV [kW] \\
\hline$P_{\max }^{q}$ & Maximum charging power during transaction $q[\mathrm{~kW}]$ \\
\hline $\mathrm{T}_{\mathrm{DUR}, \text { charge }}^{\mathrm{q}}$ & The duration for which the EV is charged during transaction $q[\mathrm{~h}]$ \\
\hline $\mathrm{t}_{\text {end-charge }}^{\mathrm{q}}$ & The time at which the EV has completed the charging process for transaction $q[-]$ \\
\hline $\mathrm{C}_{\text {parking }}$ & Parking area capacity [-] \\
\hline $\mathrm{S}$ & Apparent power [kVA] \\
\hline $\mathrm{P}$ & Active power [kW] \\
\hline $\mathrm{R}_{\text {peak,transf. }}$ & Relative peak load [-] \\
\hline$P_{\text {transf. }}$ & Power experienced by transformer [kW] \\
\hline $\mathrm{C}_{\text {transf. }}$ & Rated capacity of a transformer kW] \\
\hline $\mathrm{P}_{\mathrm{av}}^{\mathrm{q}}$ & Average charging power during transaction $q[\mathrm{~kW}]$ \\
\hline$f_{\text {daily,av }}$ & Average daily transaction frequency of an EV [EV/day] \\
\hline $\mathrm{E}_{\text {daily,av }}$ & Average daily charged energy for an EV [kWh/EV/day] \\
\hline $\mathrm{N}_{\mathrm{EV} \text {,groups }}$ & Number of EV groups assigned to a transformer [-] \\
\hline $\mathrm{N}_{\mathrm{CS}}$ & Number of charging stations [-] \\
\hline$\Delta \mathrm{T}_{\text {flex }}$ & Available flexibility [h] \\
\hline \multicolumn{2}{|l|}{ Acronyms } \\
\hline EV & Electric vehicle \\
\hline RES & Renewable energy resources \\
\hline LV & Low-voltage \\
\hline PV & Photovoltaics \\
\hline BEV & Battery electric vehicle \\
\hline MV & Medium-voltage \\
\hline CS & Charging station \\
\hline EVID & A unique anonymous identity for each EV $j$ that occurs in the dataset \\
\hline PF & Power factor \\
\hline USP & Utrecht Science Park \\
\hline $\mathrm{P} \& \mathrm{R}$ & Park\&Ride \\
\hline ZEV & Zero-emission vehicle \\
\hline FCEV & Fuel cell electric vehicle \\
\hline V2G & Vehicle-to-grid \\
\hline DSO & Distribution system operator \\
\hline MaaS & Mobility as a service \\
\hline
\end{tabular}

reinforce local distribution grids in some locations for an effective integration of EVs.

Other work that analysed the impact of EVs on the distribution grid are [11,12]. This paper differs from these works on some aspects. While Ramanujam et al. [12] examines a similar case, their simulations are driven by synthetic estimates, rather than real-life empirical data, therefore being a less accurate characterisation of real-life conditions. This paper uses a real EV transaction data set which yields more realistic results. Verzijlbergh et al. [11] examines the distribution grid impacts using charging profiles based on real life driving data, as done in this paper. However, the study is mainly applicable to the system level, rather than for individual network components, as achieved in this paper.

The academic field has proposed several intelligent or smart EV charging management methods to handle the potential problems described. Ayyadi et al. [13] recommended tariff based charging in a residential area. Di Silvestre et al. [14] used an optimisation approach to devise efficient management strategies for 
EV parking lots. A study by Sehar et al. [15] showed the impacts of plug-in EVs on a retail's building's peak demand energy consumption and presented the ability of renewable energy resources (RES) and demand management options to reduce their impacts. Sehar et al. [15] concluded that $38 \%$ of the EV load demand could be absorbed by demand management in combination with photovoltaics (PV). Huls et al. [7] proposed coordinated charging strategies for plug-in EVs to facilitate a flexible charging process that may be delayed in time, ensuring that the user's charging requirements does not suffer from the utilized flexibility. Pirouzi et al. [16] proposed a model for power management in a smart distribution network in which the objective is to minimise the costs of energy and improve the voltage profile. The general conclusion from these studies is that the existing distribution grid should be able to accommodate a substantial penetration level of EVs if the majority of the charging demand is controlled. Uncontrolled charging of EVs coinciding with the peak hours of residential demand is expected to lead to component overloading and excessive voltage deviations [17].

Previous studies have been focused on the flexibility of EV demand and controlled charging [18-21]. Here, the flexibility of EV demand is defined as the difference between the connection duration and the charging duration. The studies [18-21] looked at residential charging, instead of office charging, as done in this paper. Only a limited number of studies have investigated the EV charging demand in office areas. In an office area, the EV charging demand is likely to differ from a residential area. In case of uncontrolled charging, the EV demand peaks are likely to coincide with peak hours of economic activity as employees plug-in around the same time, for example at the beginning of the work day. Furthermore, the EV charging demand is likely to be concentrated in the same areas, i.e. parking areas. As the existing distribution network is rated to deliver electricity depending on historical electricity demand [17], increased EV demand in these areas might introduce grid congestion. Grid congestion can be avoided by redistribution of the EV charging demand over the period that the $\mathrm{EV}$ is connected to the charging station, e.g. smart charging. Furthermore, smart charging can provide ancillary services, minimise charging costs and optimal utilisation of renewable energy generation [22].

Prior work likewise focused on EV session data gathering and analysis. Sadeghianpourhamami et al. [23] provided a comprehensive analysis and quantification measures of flexibility characteristics using actual data from 390,000 EV charging sessions in The Netherlands. A coordinated charging optimisation algorithm is developed to assess the flexibility exploitation for load flattening and load balancing with renewable generation. The concept of the work in [23] is similar to our paper. It likewise focuses on flexibility analysis and quantification using real transaction data from The Netherlands. It is related to the methods and the results of our paper. However, there is no grid impact assessment or simulation of future EV charging transactions. Furthermore, office areas are not distinguished from residential areas. Flamini et al. [24] analysed the charging variables, such as connection time, charging duration, and charged energy, using an actual dataset of 400,000 EV charging transactions in the Netherlands, in order to understand the EV drivers charging behaviour. A statistical characterisation methodology was developed to represent the multimodal probability distributions of the charging variables. The work in [24] is related to our study as the authors calculate and analyse similar metrics such as the connection time, plugin and plug-out time and the EV power demand, however they did not assess the future EV demand and the possible mitigation by EV demand flexibility or quantify the transformer peak load. Furthermore, office areas are not distinguished from residential areas and no clear results on the flexibility potential are presented as in our paper.
The primary goal of our study is to determine the possible increase in load due to EVs charging in an office area, analyse the impact on the load experienced by existing distribution transformers and identify when the grid transformers become overloaded. The study is empirical and is based on analysing real transaction data, in comparison to some of the studies mentioned before. An additional goal is to evaluate the time-dependent flexibility of the EV demand and the mitigation of the load. The mitigation of the load is done by applying controlled charging. In the controlled charging scenario, the load is spread out over the connection time of the EV based on a heuristic approach. In contrast to the papers that studied residential charging, this paper focuses on office charging. The impact of an office fleet consisting entirely out of battery electric vehicle (BEVs) charging on the distribution network of an office area is analysed, which is in line with the Dutch government's ambition and goals for 2050. The impact and time-dependent flexibility is determined at the MV/LV transformer level and a mitigation strategy is defined. The proposed methods are generic and can be applied to assess the grid impact of future EV charging demand in different areas, whereas the case study demonstrates their application. The methods are replicable and can be applied to other case studies likewise. The conclusions are mainly specific for the case study, but we conclude on a general note that the placement of CS should be strategically performed looking at the capacity of the network and taking into account the forecasted load due to EVs charging.

The contribution of this paper can be summarised as follows:

- A method is proposed for determining the grid impact of future EV charging demand.

- The time-dependent flexibility is determined and a mitigation strategy is proposed.

- An empirical study for grid impact assessment, flexibility determination and mitigation strategy for a case study addressing an office area in The Netherlands.

The paper is structured as follows. In Section 2 the methods are described. The proposed methods are applied to a case study, which is described together with the data inputs in Section 3. The simulation results, impact calculation results and flexibility results are presented in Section 4. The paper is concluded with discussion in Section 5 and concluding remarks in Section 6.

\section{Methodology}

This section describes the methods used to create power demand profiles of simulated EVs and determination of EV flexibility. To give a representation of real-life EV charging behaviour, this method makes use of a dataset including real EV charge transaction data. Herein, first the method used to create power demand profiles of simulated EVs is described in Section 2.2. In Section 2.3 the method for determining the impact on MV/LV transformers is explained. Section 2.4 will provide the method for a flexibility assessment and a proposed mitigation.

In the proposed method, for each EV transaction $q$, the following data is required:

$t_{\text {plug-in }}^{q}$ : the plug-in time for transaction $q$.

$t_{\text {plug-out }}^{q}$ : the plug-out time for transaction $q$.

$E_{\text {req }}^{q}[k W h]$ : the total charged energy during each transaction $q$. EVID: A unique anonymous identity for each $\mathrm{EV} j$ that occurs in the dataset. 


\subsection{EV fleet size}

As this study looks into the charging demand in an office area, two EV categories are distinguished, namely a company EV and a commuting EV. The company EV is shared among the employees in the office area. The commuting EV is owned or leased privately by employees. An EV is assumed to be a company $\mathrm{EV}$ when the maximum duration of its connection to a charging station (CS) has been more than $24 \mathrm{~h}\left(H_{\text {DUR,max }}>24 \mathrm{~h}\right)$ and it has started the charging process at least once in the evening hours ( $\left.H_{\text {plug-in,max }}>16: 00\right)$, otherwise it is considered a commuting EV. The categorisation is illustrated in Fig. 1.

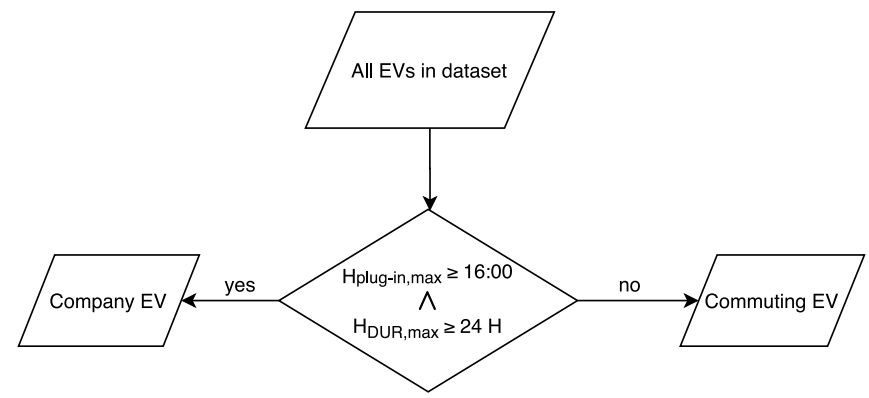

Fig. 1. Categorisation of EVs in the logged dataset.

To study the impact of an EV fleet charging in the office area assumptions regarding the size and penetration rate are made, as follows:

- The company vehicle is considered a BEV [25].

- All passenger vehicles are assumed to be BEVs, based on the Dutch ambition for 2050 [4]. Therefore, all EVs should be either a company BEV or a commuting BEV.

- The share of company EVs on the EV fleet is determined at $28 \%$, based on a linear interpolation on data from [26]. The corresponding share of commuting BEVs is $72 \%$ for the year 2050.

- Evaluating the modal split and the number of daily visitors [27], 9699 daily used passenger vehicles are estimated in the present day.

- The growth of the passenger vehicle fleet in the studied area is assumed to be equal to that of the Dutch passenger vehicle fleet $(0.6 \%)$ [28].

- The EV fleet at the investigated area is therefore sized at around 11,724 EVs in $2050\left(N_{E V, 2050}\right)$.

The charging profile of an EV depends on the category of the EV, the power at which the EV charges and the frequency at which it needs to charge. The EV dataset is analysed and histograms of the plug-in hour of the day $\left(H_{\text {plug-in }}[\mathrm{h}]\right)$, the total charged energy $\left(E_{\text {req }}[\mathrm{kWh}]\right)$ and the connection time $\left(T_{\text {connect }}[\mathrm{h}]\right)$ are created, following the method described in [20].

\subsection{EV charging profiles stimulation}

For the method of the simulation of the EV charging profiles we adopt the method by Gerritsma et al. [20]. In [20], a transaction specific charging power dataset was used to generate EV future charging demand profiles. However, in the data used in our study, transaction specific charging power was not available. Therefore we assume that the EV charges at a maximum charging power, $P_{\text {Max }}$, of $22 \mathrm{~kW}$, based on the nominal power of the logged CSs, see Section 3.

The adaptation of the method by [20] for the charging profile simulation is as follows: For each transaction, an EV number is assigned, depending on the charge frequency. As each EV has been assigned a category in representing the EV fleet, the volume the EV is charged with during transaction $q$ is randomly picked from a list which contains the volumes and certain probabilities for that EV category. Per transaction $h_{\text {plug-in }}^{q}$ is assigned, based on the probability an EV of a certain category plugs-in at that hour. The exact simulated plug-in time, $t_{\text {plug-in }}^{q}$, is derived by assigning a number of minutes within $h_{p l u g-i n}^{q}$. With the number of minutes randomly chosen. It is assumed that all EVs in the simulation charge uncontrolled at power rate $P_{\text {Max }}^{q}$, starting at $t_{\text {plug-in }}^{q}$ and ending when $E_{\text {req }}^{q}$ of that specific transaction is reached. The duration the EV is connected to the CS, $T_{\text {connect }}$, is selected randomly based on the EV's category and the probability of the connected hours for that EV category. The dependency of $E_{\text {req }}^{q}$ and $\Delta T_{\text {connect }}^{q}$ are respected by randomly choosing $T_{\text {connect }}$ based on the simulated $E_{\text {req }}$. This means that a transaction with a large required energy, has a high probability of a longer connection time. The plug-out time, $t_{\text {plug-out }}^{q}$ is then determined by summing $t_{\text {plug-in }}^{q}$ with $T_{\text {connect }}^{q}$. The duration for which the EV is getting charged is determined by dividing the volume charged by the power at which it charges as expressed in Eq. (1). The time at which the EV has completed the charging process, $t_{\text {end-charge }}^{q}$, is then determined by summing $t_{\text {plug-in }}^{q}$ with $T_{\text {DUR,charge }}^{q}$.

$T_{\text {DUR, charge }}^{q}=E_{\text {req }}^{q} / P_{\text {max }}^{q}$

\subsection{Impact of charging demand on distribution grid transformers}

In this section a grid impact calculation provides insight into the effect of EVs charging on distribution transformers. Aggregation of EV demand profiles is applied in order to reduce the computational burden. The charging profiles of the EV fleet are clustered into 20 groups, as done by [29], which makes it computationally feasible. The number of clustered groups was chosen in a heuristic manner. The logic behind this choice is that larger number of groups would result in longer computation times, whereas a smaller number of groups would make the number of CS too large to spread them out over the parking areas of the studied area. Then the MV/LV transformers on which the charging load is expected are selected based on the parking areas, as follows:

1. The parking areas and their parking capacities in the area are defined;

2. Parking areas without sufficient parking capacity $\left(C_{\text {parking }}\right)$ for at least one group are excluded;

3. Parking areas without a MV/LV transformer close by are excluded.

For this method, the base load of the transformers in the studied area is necessary. The extra load due to EV charging demand is then added on top of the base load.

The load of a transformer is characterised by its seasonal variation, with two seasonal demand periods - winter and summer. Therefore, one week in each seasonal period is analysed. Note that since the rated capacity of a transformer is apparent power (S) [kVA], in the analysis an average power factor (PF) is used to convert the apparent power to active power $(\mathrm{P})[\mathrm{kW}]$, according to Eq. (2). Here, $0 \leq P F \leq 1$ is the power factor. For the analysis, the average power factor of 0.9 and 0.95 is used for summer and winter, respectively, which represents the average power factor in these seasons [30]. The variable power factor is attributed to the temperature difference between summer and winter which influence the power quality of the transformer.

$P=S * P F$

The relative peak load of the transformers are studied. As a transformer allows to be overloaded for some time, the overload 
criterion is 1.25 , and the critically overload criterion considered is 1.5 , as considered in [30]. The relative peak load ( $\left.R_{\text {peak,transf. }}\right)$ experienced by a transformer is determined by dividing the power the transformer experiences $\left(P_{\text {transf. }}\right)$ by the rated capacity of the transformer ( $\left.C_{\text {transf. }}\right)$, according to Eq. (3).

$R_{\text {peak, load,transf. }}=P_{\text {transf. }} / C_{\text {transf. }}$

To allocate which part of the peak load is due to the integration of EVs, the peak load experienced by the transformers is also determined for a scenario without EV. The scenario without EV does not include charging profiles of EVs, solely an annual load growth of $1.5 \%$ is considered in this scenario.

\subsection{Mitigation of impact by demand flexibility}

In Section 2.3 the impact of uncontrolled charging of a vehicle fleet consisting solely out of BEVs on MV/LV transformers is investigated. This section describes the analysis of the time-dependent flexibility of EV demand and the method for controlled slow charging of EVs. The method to generate the time-dependent flexibility of EV demand is obtained from Gerritsma et al. [20]. The results in [20] confirm the feasibility of congestion management using smart charging within flexibility constraints in residential areas. The method used in this paper provides an answer to the available flexibility of EV demand on $\mathrm{MV} / \mathrm{LV}$ transformers in office areas.

In Section 2.2 charging profiles of uncontrolled charging are generated. With uncontrolled charging it is assumed that an EV is charged at $P_{\max }^{q}$ when that EV is plugged in. However, the charging power might be slowed down to an average charging power $P_{a v}^{q}$ $[\mathrm{kW}]$, i.e., controlled charging. In this study the following approach has been followed for determining the controlled charging scenario: with controlled charging it is assumed that $E_{\text {req }}^{q}[\mathrm{kWh}]$ for the transaction $q$ has to be met by the time the EV is pluggedout. We assume that the charging session is spread out over the whole period the EV is plugged-in. This charging method results in a different simulated charging power profile because the EV charges with a lower charge rate, $P_{a v}^{q}$, over the connection time, $\Delta T_{\text {connect }}^{q}$. Per transaction, $P_{a v}^{q}$ is determined by using Eq. (4). The before-mentioned simulation steps are carried out for each $\mathrm{EV} j$.

$P_{a v}^{q}=E_{\text {req }}^{q} / \Delta T_{\text {connect }}^{q}$

\section{Distribution network case study and data collection}

In this paper the distribution grid of Utrecht Science Park (USP) has been considered as a case study. This area is located on the east side of the city of Utrecht, the Netherlands. This is an area for education, research, entrepreneurship and healthcare. USP includes 108 companies, 2500 student houses, 51,000 students and approximately 26,000 employees [27]. This area is considered an office area and therefore this study looks into office charging only, while residential charging is excluded.

Since this study seeks to understand the impact of EVs, a transaction dataset of actual EV charging is used. The transaction dataset consists of the charge data of EVs at 4 logged CSs in the studied area. Last Mile Solutions is the supplier of the CSs and keeps track of the transactions that occur at the CSs. The CSs were logged from the January 2, 2017 up to and including February 28, 2019. Each CS is equipped with two three-phase charging points at $32 \mathrm{~A}$, with connection in a $230 \mathrm{~V}$ network, hence each charging point can provide a three-phase charging power of $22 \mathrm{~kW}\left(P_{\max }\right)$. The dataset includes detailed information per transaction such as the plug-in time, the plug-out time, the total charged energy and an anonymous ID for each EV.

Data cleaning steps included removing transactions where no EV ID was logged. Furthermore, the dataset was filtered so that

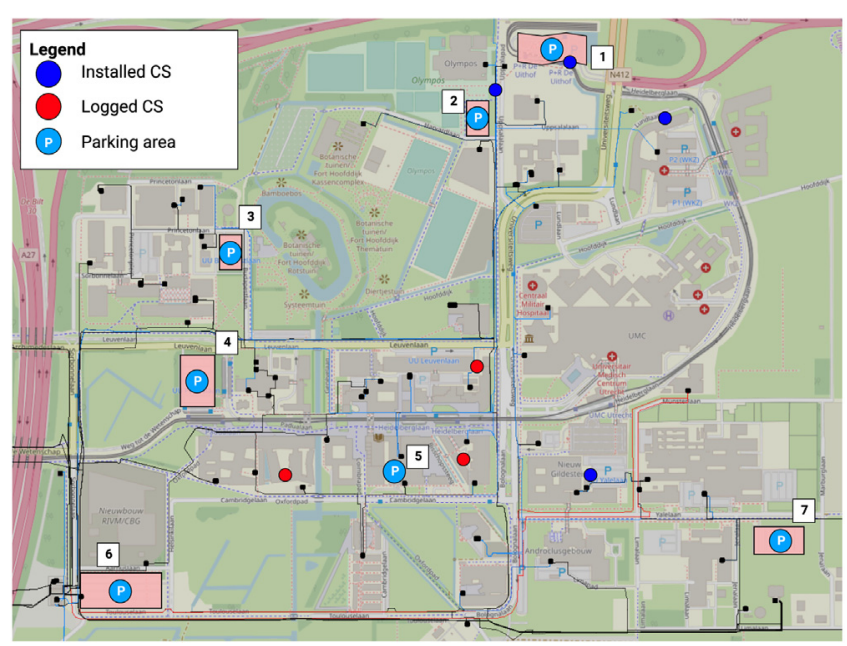

Fig. 2. Representation of the distribution network and locations of CSs at USP.

Table 1

Key results of EVs charging at CSs at Utrecht Science Park, based on actual measurements within the period from February 28, 2018 to February 28, 2019

\begin{tabular}{llll}
\hline Category & No. of EVs & $\begin{array}{l}f_{\text {daily,av }} \\
{[\mathrm{EV} / \text { day }]}\end{array}$ & $\begin{array}{l}E_{\text {daily,av }} \\
{[\mathrm{kWh} / \mathrm{EV} / \text { day }]}\end{array}$ \\
\hline Company EV & 4 & 0.412 & 4.874 \\
Commuting EV & 18 & 0.027 & 0.548 \\
All EVs & 42 & 0.053 & 0.708 \\
\hline
\end{tabular}

it covers a one-year period, the start date was February 28, 2018 and the end date February 28, 2019. This resulted in a dataset of 807 transactions.

At the time of writing this paper, there are 21 CSs installed at the 7 locations shown in Fig. 2, of which 4 were logged. The logged CSs are displayed with a red dot in Fig. 2.

\section{Results}

\subsection{Results of measured EV data set}

The key results determined from the measured data set are shown in Table 1. As can be observed by the daily energy demand, company EVs have a high impact on the aggregated electricity demand in comparison to the other EV categories. The company EVs have the highest charge frequency, with an average charge frequency of 0.412 [EV/day].

The distribution of several parameters was analysed for the different identified categories. Fig. 3 shows histograms of the four parameters analysed. The first row in the figure shows the plug-in hour per EV category. It illustrates that company EVs plug-in mostly around 10:00, commuting EVs plug-in a bit earlier with a peak between 7:00 and 8:00. The second row of graphs shows the distribution of the required energy for EVs in different categories. The distributions for company EVs and commuting EVs are quite similar. Lastly, the third row in the figure shows the connection duration, $T_{\text {connect }}$. With company EVs having the longest connection duration, followed by commuting EVs.

\subsection{Simulation of EV charging profile}

In this section, the results of the charging profile simulation of the EV fleet per scenario are described. Based on the daily used passenger vehicles in 2019 and an average growth rate of $0.6 \%$, 11,724 EVs are expected in the investigated area in 2050 [28]. 

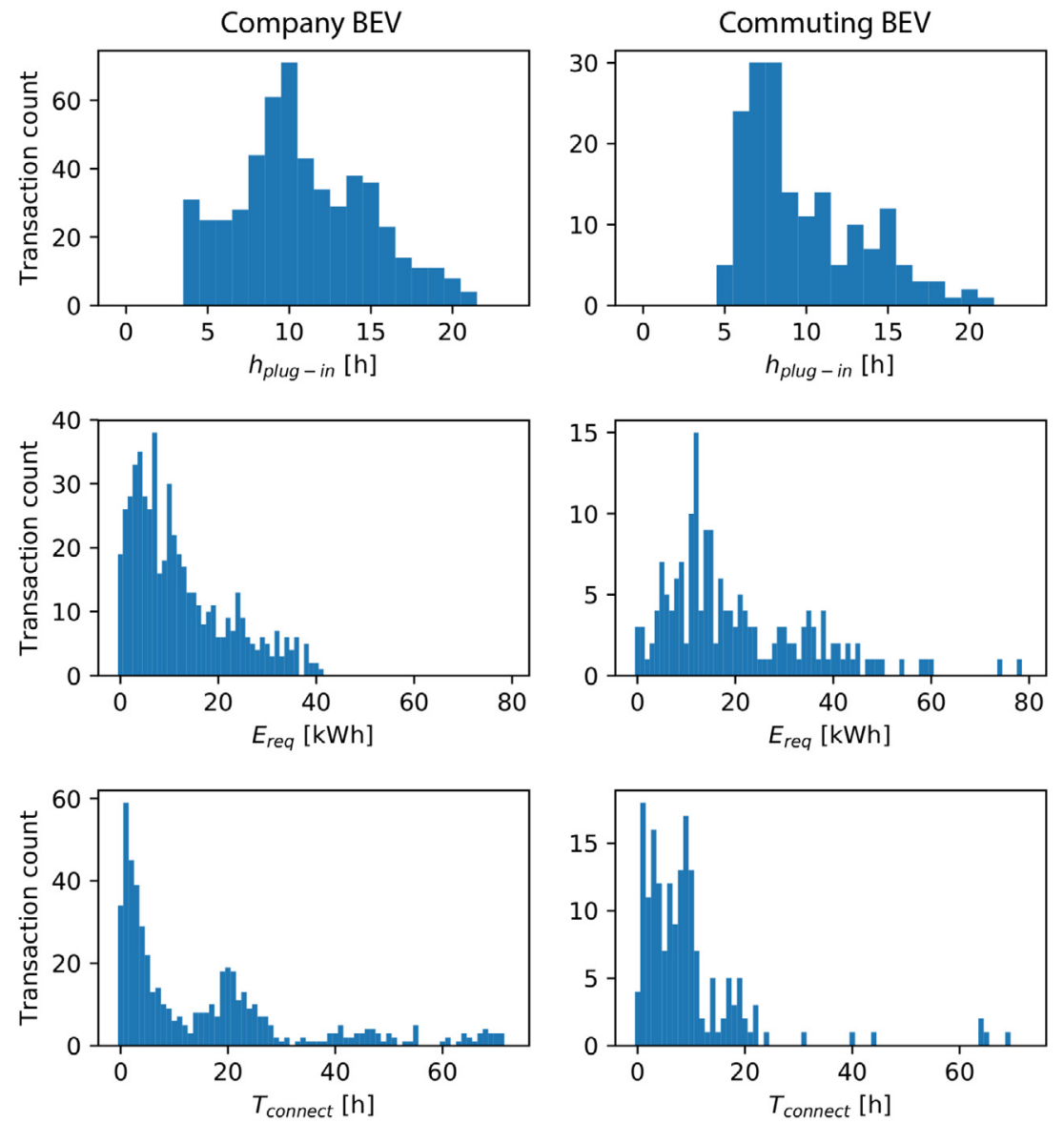

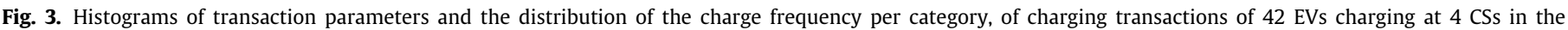
period of the logged year, February 28, 2018 to February 28, 2019.

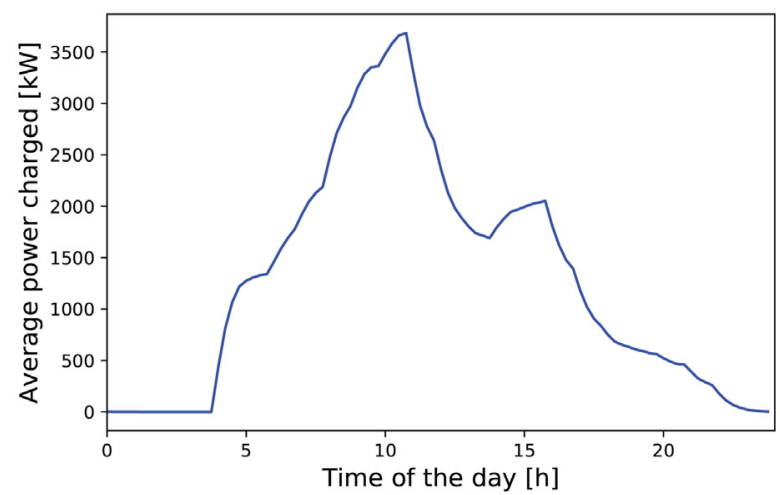

Fig. 4. Average daily charging profile for simulated EVs in the investigated area.

Therefore, 3347 company EVs and 8377 commuting EVs are simulated. The average load profile of 11,724 EVs charging at the investigated area is shown in Fig. 4. The peak power expected the simulated year is $6094 \mathrm{~kW}$ and the aggregated energy demand over the year is $11,294,701 \mathrm{kWh}$. In the period of the simulated year, a maximum of 1713 EVs are connected at once. In order to accommodate for the uncontrolled charging of the simulated number of EVs, 857 CSs should be available at the investigated area.
Table 2

Key specifications of the CS locations.

\begin{tabular}{lllll}
\hline Parking area number and location & $C_{\text {parking }}$ & $N_{\text {EVgroups }}$ & $N_{C S}$ & $C_{\text {transf. }}[M V A]$ \\
\hline 1. P\&R Utrecht Science Park & 2000 & 8 & 379 & 0.4 \\
2. Parking area P10 “Uppsalalaan" & 125 & 1 & 62 & 0.63 \\
3. Parking area P9 "Budapestlaan" & 135 & 1 & 61 & 0.63 \\
4. Parking area "Padualaan" & 419 & 2 & 98 & 0.63 \\
5. Parking garage P8 "Cambridgelaan" & 504 & 3 & 132 & 1.25 \\
6. Parking area "Sorbonnelaan" & 613 & 3 & 136 & 0.63 \\
7. Parking area P7 "Jenalaan" & 236 & 2 & 111 & 0.63 \\
\hline
\end{tabular}

\subsection{Impact of charging demand on MV/LV transformers}

Table 2 describes the main specifications of the determined possible locations for CSs including the parking area capacity $\left(C_{\text {parking }}\right)$ the number of groups assigned to the transformer $\left(N_{E V \text { groups }}\right)$, the number of CSs $\left(N_{C S}\right)$ and the transformer capacity ( $C_{\text {transf. }}$ ). The numbers in the table correspond to the numbers in Fig. 2.

Table 3 represents the expected MV/LV transformer peak load, together with the without EV-scenario, which just includes the annual load growth of $1.5 \%$. As mentioned in Section 2.3, the overload criterion is 1.25 . The overload criterion reflects the instantaneous peak value, which can be higher than the rated capacity. As we can see from the table, the transformer at Park\&Ride (P\&R) USP and the transformer at Sorbonnelaan street are critically overloaded, as their relative peak load is higher than the critically overload criterion. The transformers at P7 Jenalaan street and Padualaan street are overloaded. None of the transformers are overloaded in the without EV-scenario. 


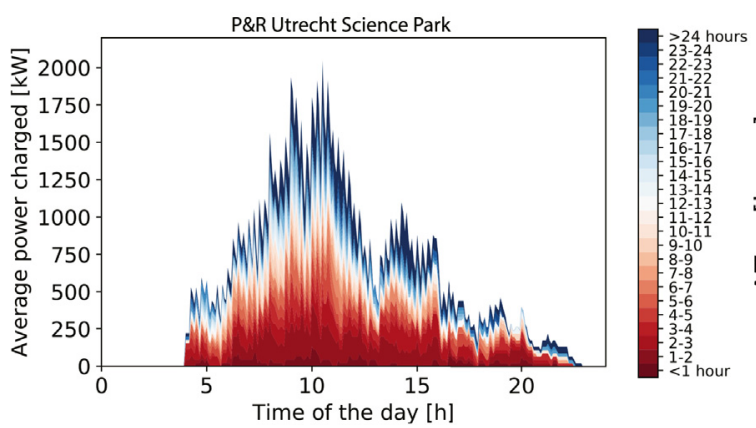

(a) Transformer P\&R Utrecht Science Park.

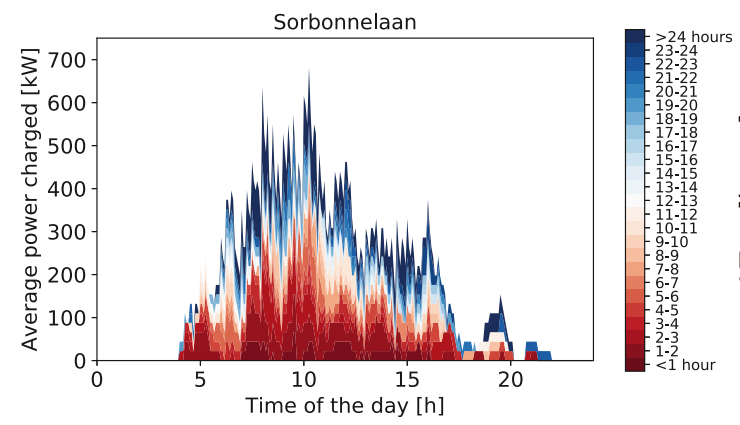

(c) Transformer Sorbonnelaan.

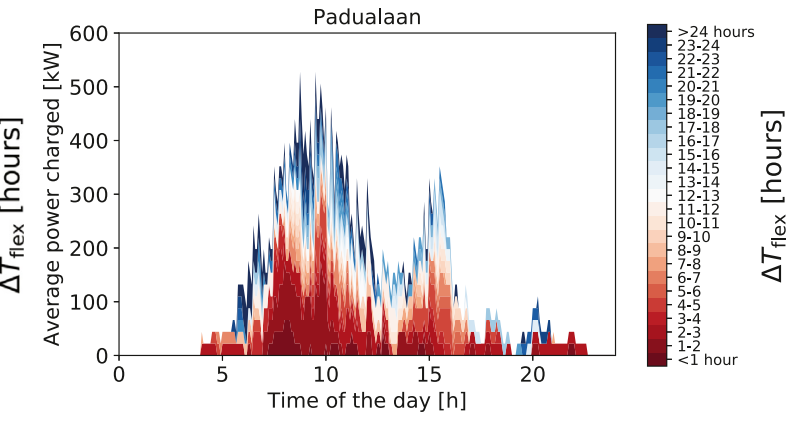

(b) Transformer Padualaan.

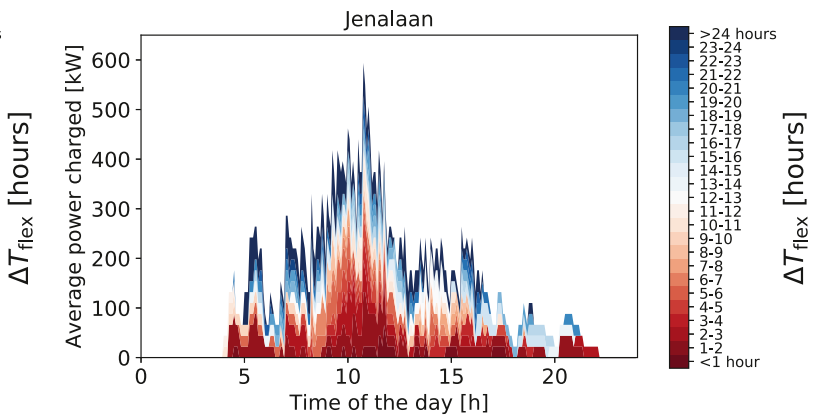

(d) Transformer Jenalaan.

Fig. 5. Available flexibility of aggregated EV demand at the transformer in different office areas.

Table 3

Expected relative MV/LV transformer peak load [-].

\begin{tabular}{lll}
\hline Parking location & EV & Without EV \\
\hline P\&R Utrecht Science Park & 6.30 & 0.52 \\
Parking area P10 “Uppsalalaan" & 0.94 & 0.68 \\
Parking area P9 "Budapestlaan" & 0.86 & 0.27 \\
Parking area "Padualaan" & 1.39 & 0.33 \\
Parking area P8 "Cambridgelaan" & 0.80 & 0.05 \\
Parking area "Sorbonnelaan" & 1.75 & 0.31 \\
Parking area P7 "Jenalaan" & 1.27 & 0.33 \\
\hline
\end{tabular}

\subsection{Flexibility of EV demand}

The time-dependent flexibility is determined for the expected overloaded transformers. To make a clear representation, the flexibility is obtained for one day in the simulated period. Fig. 5 demonstrates the charging power for the transformers at P\&R USP, Padualaan, Sorbonnelaan and Jenalaan streets, respectively. The different colours indicate the potential $\Delta T_{\text {flex }}$ [h] for different parts of the average measured aggregated EV demand starting at each time of the day. As mentioned earlier, $\Delta T_{\text {flex }}$ is defined as the number of hours over which the demand could be shifted within the connection duration of an EV. The figures indicate the opportunities to shift the EV energy demand over time, i.e., 52\% of the demand at Padualaan street can be delayed for more than $8 \mathrm{~h}$ and $9 \%$ of the demand for more than $24 \mathrm{~h}$. For Jenalaan street, $56 \%$ and $15 \%$ of the demand can be delayed for more than 8 and $24 \mathrm{~h}$, respectively. For Sorbonnelaan street this is $52 \%$ and $16 \%$, respectively. For P\&R USP this is $49 \%$ and $14 \%$, respectively.

For the overloaded transformers, the load experienced by the transformer is determined when EVs use controlled charging (in this case $E_{\text {req }}^{q}$ for the transaction is met when the EV is pluggedout). As stated in Section 4.3, expected peak power at the studied area is $6094 \mathrm{~kW}$ under uncontrolled charging. By applying slow charging, the peak power is reduced to $3297 \mathrm{~kW}$, a reduction of $54.1 \%$. Furthermore, by applying slow charging, overloading of 3

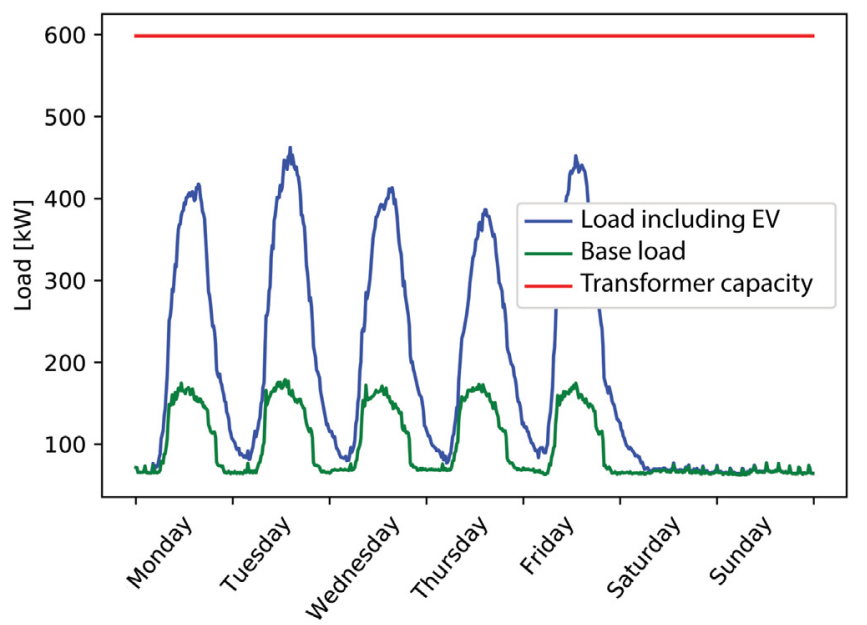

Fig. 6. Load during a week at Transformer Padualaan under controlled charging.

out of the 4 transformers can be mitigated. Critical overloading is still experienced by the transformer at P\&R USP.

An example of the load experienced by the otherwise overloaded transformer when controlled charging is put in place is displayed in Fig. 6. This example shows that overloading can be mitigated when controlled charging is applied.

\section{Discussion}

In this section, the methods and results are discussed. First of all, the development of the future EV fleet is uncertain. For instance, the growing use of vehicle sharing may take over from traditional use of the vehicles in the office area. Fully autonomous vehicles may drive this trend further, but these modal shifts have not been analysed in our model. It is expected that most of these 
vehicles are electric [25] and therefore increase the EV driving range. Furthermore, off-peak transport is likely to continue to occur during the night. This could mean that consequently, the available flexibility in the EV demand might decrease, especially during daytime. The increased daily distances travelled per car will imply reduced parking time and therefore less flexibility in EV demand and less energy storage available for grid services and/or PV self-consumption. The implications for the EV demand flexibility, which may decrease in a future system based on shared and autonomous vehicles, is recommended to be further studied in detail. Furthermore, the Dutch government has set goals based on the term Zero-Emission Vehicles (ZEV). This term also includes fuel cell electric vehicles (FCEVs). However, the BEV will most likely continue to dominate in the short- to medium-term future ZEV fleet as the FCEV technology is not yet as developed as the EV technology, and it is presumed that the lowest cost technology will be the dominant one in the near future [25,31]. In addition, the authors in [31] concluded that less energy reduction can be obtained for a ZEV fleet if replaced by FCEVs rather than a ZEV fleet consisting of EVs, making it less attractive for policy makers to invest in this technology. For the vehicle fleet size it was assumed that the modal split of commuting employees remains constant. However, the modal split is likely to change as Utrecht region is committed to facilitate the growth of USP by investing in public transport and bicycle accessibility [5]. A recommendation for further research is to use the simulation model and vary the modal split of employees commuting to determine the peak power demand and flexibility potential.

Second, this study has not looked into the impact of increasing PV penetration rates in the investigated area. Energy production by PV is likely to grow in the investigated area as PV will play an important role in the future energy supply; it is expected to supply $25 \%$ to $30 \%$ of the energy demand in the Netherlands in 2050 [32]. This means that some transformers might experience less load due to local PV power generation. Installing PV systems would supplement EVs charging in the office area. This is due to the fact that, in contrast to residential charging of EVs [20], EVs in the office area charge during the day, with the charging peak around 10:00. Either, optimally placing the PV modules so that maximum generation is in the morning or smart charging of EVs would increase PV self-consumption. Results from this study show that the flexibility of the morning peak demand, could be shifted so that the EVs get charged during the PV peak generation a few hours later. This is in contrast to residential charging, where the evening EV demand holds the most flexibility potential [20]. This means that more time-dependent flexibility would be necessary in case of residential charging as to shift the evening peak until the following mid-day. For office charging the flexibility in EV demand for the morning peak could be less as the peak would only needed to be shifted by a few hours. This means that EV-based smart charging can be a crucial factor to scale up variable PV power generation. Gerritsma et al. [20] addressed the fact that for smart charging, the EV users would have to actively participate and willingly share information. This is likewise the case for office charging. However, the controlled charging of EVs in an office area could be easier compared to a residential area. In the office area, especially the commuting EVs are to some extent restricted to office hours, therefore it is easier to control when the EV charges and when the charging process has to be finished (e.g. at the end of the work-day). Regarding the company EVs, which are operated by the university and local businesses, controlling the charging process can be done by EV fleet management. EV fleet management is already commercially conducted. In a residential area, charging behaviour is somewhat more unpredictable and it is therefore more difficult to control the charging process of large numbers of EVs.
Lastly, regarding the method for the creation of charging profiles, prior work has also analysed the impact of EVs on the distribution grid [11,12]. However, as discussed in Section 1, this paper differs from these works as we have made use of a real EV transaction dataset which yields more realistic results. This paper adopted a method by Gerritsma et al. [20] for the creation of charging profiles and to determine the flexibility of EV demand, but expands on this work by conducting an in-depth analysis of existing transformers in an office area and quantifies their different load profiles.

In the simulation for the creation of charging profiles weekend days were not taken into account. This is due to the fact that in the original transaction data, no transactions were identified on the weekend days. This makes sense as the investigated area is in an office area with low economic activity in the weekends. Yet, as the city of Utrecht is to expand, possibly vehicles from outside of the office area will start to charge on weekend days in this area in the future. In addition, residential buildings rising in the area will likewise change the charging behaviour of the EV fleet. The EV demand in the weekend is more likely to be spread throughout the day as these vehicles are not restricted to office hours. Therefore a lower peak demand than on weekend days can be expected, making the analysis on weekdays in this study a nonetheless relevant case.

The number of EVs charging simultaneously is left unconstrained in the simulation. This means that the number of available CSs is driven by the EV demand and the connection duration. To determine the number of necessary CSs, the maximum number of EVs connected simultaneously was taken into account. However, this maximum number of EVs connected may occur once a year. Therefore in real-life conditions the number of CSs available could be lower. In case of less available CSs in the area, lower charging peaks can be expected. However, with more available CSs, and therefore increasing EV connection periods, more flexibility in EV demand can be expected. Alternately the actual charging time of an EV could be used to determine the number of CSs required. It would be interesting to determine the maximum number of EVs charging simultaneously. The way EVs are charged in the future may look different from the current situation. A mechanism could be put in place that automatically disconnects an EV when its fully charged and connects the following EV. This is however speculative and not in place as of writing this paper.

As the original transaction data did not include the transaction specific charging power, a fixed charging power was set to 22 $\mathrm{kW}$ for EVs, based on the nominal power of the logged CSs. This is not a rare assumption as a fixed charging power was set by several other studies such as $[11,20,33,34]$. The charging power might actually be lower than the maximum charging power, but no comparison could be made with the original transaction data. This is due to the fact that in the EV transaction dataset used, no charging power was logged. If the original transaction data actually included transaction specific charging power, a more accurate charging profile could be created. In case that EVs actually charge with a lower power, the EV peak power and the flexibility in EV demand would be overestimated. However, in the future the charging power of EVs is likely to be driven to higher power rates [35]. A recommendation for further research is to use the simulation model with varying the charging power when simulating the charging profiles for determining the peak power demand and flexibility potential.

In Section 4.4, we showed what would happen if use is made of the time dependent flexibility in EV demand. Solely by spreading out the EV demand over the connection period, the peak load of EV demand in the studied area was reduced by $54 \%$. In this case study, overloading could be mitigated for 3 out of the 4 overloaded transformers. The mitigation of the charging load is 
done by applying controlled charging. In the controlled charging scenario, the load is spread out heuristically over the connection time of the EV. However, this might not be the optimal mitigation strategy. An optimisation approach should be performed in order to determine the lowest peak power possible in the studied area. Furthermore, other smart charging strategies such as V2G, tariffbased charging and load shifting and their mitigation on grid impact are recommended to be explored for this case study in future research.

The proposed methods in this paper are applied for the case study the USP, however these methods are replicable and can therefore be applied to other case studies likewise. For example, a city or a country assessment can be performed to subsequently determine the bottlenecks in the distribution grid and obtain the flexibility of EV demand in those areas. Such an assessment is valuable as it could be input to the distribution system operators (DSO) investment decision making in grid reinforcement. For DSOs to achieve an effective and timely grid reinforcement, it is key to know which locations are expected to receive the highest load due to EV charging and which part of the EV demand cannot be decreased by controlled charging. Other stakeholders can benefit from the proposed methods. For example, municipalities can apply the methods proposed in this paper to strategically place CSs, looking at the capacity of the network and taking into account the forecasted energy demand due to EVs charging.

\section{Conclusion}

This paper presents a methodology to determine the impact of an office car fleet consisting entirely out of BEVs charging at the distribution network of an office area. Furthermore, the time-dependent flexibility is determined at the MV/LV transformer level and a mitigation strategy is proposed. The proposed methodology is generic in the sense that it can be applied to assess the grid impact of future EV charging demand in different areas, whereas the investigated case study elucidates its application. A scenario was created for the size of the future EV fleet at USP that complies with the Dutch government's ambition and goals for the Dutch passenger fleet. Then the charging profiles of the simulated EV fleet were created. The EV fleet in the studied area is estimated at 11,724 in 2050 . This EV fleet may result in a peak power demand of about $6 \mathrm{MW}$.

The simulated average daily charging profile exhibits a peak demand at around 10:00 and a lower peak later in the afternoon, around 16:00. In order to accommodate the expected EV fleet charging, the number of CSs necessary at the studied area are 857 in 2050. Simulations were carried out to determine the impact of EV load on the MV/LV transformers in two scenarios (2050 and without EV). The results showed that no overloading occurs in the scenario without EV. In the 2050 scenario, 4 out of 7 investigated transformers become overloaded and with no mitigation strategy put in place, these transformers are to be replaced with transformers of a higher nominal capacity. The mitigation of the grid impact is elaborated upon. It was also shown that part of the peak demand experienced at certain transformers can be delayed. This flexibility in EV demand is elaborated on by determining the charging profiles of the simulated EV fleet when controlled slow charging is applied. By applying slow charging, the charging peak can be reduced by $54.1 \%$. Overloading of 3 out of the 4 transformers can be mitigated. Critical overloading is still experienced by the transformer at P\&R USP, this is due to the fact that the existing transformer has a small nominal capacity (400 kVA) and that most EVs are expected to charge at this location because of the high parking capacity. The current small nominal capacity gives the impression that the transformer was installed at the P\&R without taking into account the increasing load due to EV charging. When controlled slow charging is applied, the transformer experiences a peak demand of around 1.2 MW. In this case, the nominal capacity should at least be about $1.2 \mathrm{MW}$, three times the current nominal capacity. Further research into other mitigation strategies can provide a better view into the transformer capacity requirements for this case study.

Overall, the results are representative for other office areas, in the sense of timing of the economic activity and work schedules in an office environment. However, it is important to note that the results are largely based on (a) dataset of 807 transactions over one year, which is rather limiting due to the early adoption of EVs in the studied area, and (b) it is based on assumptions about the future EV fleet and share between company and commuting EVs, which might change in the future as new Mobility as a Service (MaaS) business models are introduced. Still, all assumptions are supported by statistical data and/or the results of other scholars, and are presented in a transparent manner in support of future research.

In conclusion, placement of CS should be strategically performed, looking at the capacity of the network and taking into account the forecasted load due to EVs charging. Placement of CSs where grid capacity is available and controlling the charging process could avoid grid congestion and subsequently postpone and or avoid grid reinforcement. This consequently could mean a decrease in the costs of energy and an effective integration of EVs in office areas.

\section{CRediT authorship contribution statement}

M.A. van den Berg: Conceptualization, Methodology, Software, Formal analysis, Investigation, Visualization, Writing - original draft. I. Lampropoulos: Supervision, Conceptualization, Validation, Writing - review \& editing. T.A. AlSkaif: Supervision, Validation, Writing - review \& editing.

\section{Declaration of competing interest}

The authors declare that they have no known competing financial interests or personal relationships that could have appeared to influence the work reported in this paper.

\section{Acknowledgements}

We would like to thank W.L. Schram, M.K. Gerritsma and N.B.G. Brinkel for their support in the research design and their contributions in computer simulations. This study was supported by the European Regional Development Fund (ERDF) "EFRO Kansen voor West II" through the project "Smart Solar Charging regio Utrecht".

\section{References}

[1] Z. Wang, L. Yang, Delinking indicators on regional industry development and carbon emissions: Beijing-Tianjin-Hebei economic band case, Ecol. Indic. 48 (2015) 41-48, http://dx.doi.org/10.1016/J.ECOLIND. 2014.07.035, URL https://www.sciencedirect.com/science/article/pii/ S1470160X14003446?via\{\%\}3Dihub.

[2] J.L. Schnell, V. Naik, L.W. Horowitz, F. Paulot, P. Ginoux, M. Zhao, D.E. Horton, Air quality impacts from the electrification of light-duty passenger vehicles in the United States, Atmos. Environ. 208 (2019) 95-102.

[3] I. Lampropoulos, T. Alskaif, W. Schram, E. Bontekoe, S. Coccato, W. van Sark, Review of energy in the built environment, Smart Cities 3 (2) (2020) $248-287$.

[4] RVO, Statistics electric vehicles in the Netherlands (up to and including July 2020), 2020, pp. 1-9.

[5] E. van der Waard, A. Meijles, Clean Transport Action Plan (2015-2020). Municipality of Utrecht.

[6] J.A. Lopes, F.J. Soares, P.M. Almeida, Integration of electric vehicles in the electric power system, Proc. IEEE 99 (1) (2011) 168-183, http://dx.doi.org/ 10.1109/JPROC.2010.2066250. 
[7] J. Hüls, A. Remke, Coordinated charging strategies for plug-in electric vehicles to ensure a robust charging process, in: Proceedings of the 10th EAI International Conference on Performance Evaluation Methodologies and Tools, ACM, 2017, pp. 19-22, http://dx.doi.org/10.4108/eai.25-102016.2266997, URL http://eudl.eu/doi/10.4108/eai.25-10-2016.2266997.

[8] I. Lampropoulos, E. Veldman, W.L. Kling, M. Gibescu, J.G. Slootweg, Electric vehicles integration within low voltage electricity networks \& possibilities for distribution energy loss reduction, in: Proc. 2010 Innovation for Sustainable Production (i-SUP), Sustainable Energy Conf.(3), (July) 2010, pp. 1-5, http://dx.doi.org/10.1016/00406031(77)80004-X, URL http://www.smartgridcontest.com/attachments/ idea\{_\}835\{_\}118\{_\}1308070088.5252\{_\}[2].pdf.

[9] S. Deb, K. Tammi, K. Kalita, P. Mahanta, Impact of electric vehicle charging station load on distribution network, Energies 11 (1) (2018) 1-25, http: //dx.doi.org/10.3390/en11010178.

[10] Yu Zhang, Xiaohui Song, Fei Gao, Jianfang Li, Research of voltage stability analysis method in distribution power system with plug-in electric vehicle, in: 2016 IEEE PES Asia-Pacific Power and Energy Engineering Conference, APPEEC, IEEE, 2016, pp. 1501-1507, http://dx.doi.org/10.1109/ APPEEC.2016.7779740, URL http://ieeexplore.ieee.org/document/7779740/.

[11] R.A. Verzijlbergh, M.O. Grond, Z. Lukszo, J.G. Slootweg, M.D. Ilic, Network impacts and cost savings of controlled EV charging, IEEE Trans. Smart Grid 3 (3) (2012) 1203-1212, http://dx.doi.org/10.1109/TSG.2012.2190307.

[12] A. Ramanujam, P. Sankaranarayanan, A. Vasan, R. Jayaprakash, V. Sarangan, A. Sivasubramaniam, Qantifying the impact of electric vehicles on the electric grid: A simulation based case-study, in: Proceedings of the Eighth International Conference on Future Energy Systems, ACM, 2017, pp. 228-233.

[13] S. Ayyadi, H. Bilil, M. Maaroufi, Optimal charging of electric vehicles in residential area, Sustain. Energy Grids Netw. 19 (2019) 100240.

[14] M.L. Di Silvestre, E. Riva Sanseverino, G. Zizzo, G. Graditi, An optimization approach for efficient management of EV parking lots with batteries recharging facilities, J. Ambient Intell. Humaniz. Comput. 4 (6) (2013) 641-649, http://dx.doi.org/10.1007/s12652-013-0174-y, URL http: //link.springer.com/10.1007/s12652-013-0174-y.

[15] F. Sehar, M. Pipattanasomporn, S. Rahman, Demand management to mitigate impacts of plug-in electric vehicle fast charge in buildings with renewables, Energy 120 (2017) 642-651, http://dx.doi.org/10.1016/ J.ENERGY.2016.11.118, URL https://www.sciencedirect.com/science/article/ pii/S036054421631773X.

[16] S. Pirouzi, M.A. Latify, G.R. Yousefi, Conjugate active and reactive power management in a smart distribution network through electric vehicles: A mixed integer-linear programming model, Sustain. Energy Grids Netw. (2020) 100344.

[17] P. Richardson, D. Flynn, A. Keane, Optimal charging of electric vehicles in low-voltage distribution systems, IEEE Trans. Power Syst. 27 (1) (2012) 268-279, http://dx.doi.org/10.1109/TPWRS.2011.2158247.

[18] H. Zhao, X. Yan, H. Ren, Quantifying flexibility of residential electric vehicle charging loads using non-intrusive load extracting algorithm in demand response, Sustainable Cities Soc. 50 (2019) 101664.

[19] H. Khalkhali, S.H. Hosseinian, Multi-class EV charging and performancebased regulation service in a residential smart parking lot, Sustain. Energy Grids Netw. (2020) 100354.
[20] M.K. Gerritsma, T.A. AlSkaif, H.A. Fidder, W.G. van Sark, Flexibility of electric vehicle demand: Analysis of measured charging data and simulation for the future, World Electr. Veh. J. 10 (1) (2019) 14.

[21] D. Fischer, A. Surmann, W. Biener, O. Selinger-Lutz, From residential electric load profiles to flexibility profiles-A stochastic bottom-up approach, Energy Build. (2020) 110133.

[22] N.N. Brinkel, M.M. Gerritsma, T.T. AlSkaif, I.I. Lampropoulos, A.A. van Voorden, H.H. Fidder, W.W. van Sark, Impact of rapid PV fluctuations on power quality in the low-voltage grid and mitigation strategies using electric vehicles, Int. J. Electr. Power Energy Syst. 118 (2020) 105741.

[23] N. Sadeghianpourhamami, N. Refa, M. Strobbe, C. Develder, Quantitive analysis of electric vehicle flexibility: A data-driven approach, Int. J. Electr. Power Energy Syst. 95 (2018) 451-462.

[24] M.G. Flammini, G. Prettico, A. Julea, G. Fulli, A. Mazza, G. Chicco, Statistical characterisation of the real transaction data gathered from electric vehicle charging stations, Electr. Power Syst. Res. 166 (2019) 136-150.

[25] DNV-GL, Energy Transition Outlook 2019: A Global and Regional Forecast to 2050, DNVGL, 2019.

[26] KPMG, The rise of electric, shared and autonomous fleets, (February) 2019, pp. 1-20.

[27] Utrecht science park - Facts \& figures, 2019, https://www. utrechtsciencepark.nl/nl/over-het-park/facts-figures. (Accessed 25 March 2019).

[28] A. De Jong, Outlook for Prosperity and Living Environment, PBL Netherlands Environmental Assessment Agency, The Hague, 2015.

[29] R. Verzijlbergh, The Power of Electric Vehicles - Exploring the Value of Flexible Electricity Demand in a Multi-Actor Context, 2013, pp. 1-211, http://dx.doi.org/10.4233/uuid:47c8faa7-94de-40fe-8be7-fccec6ee07bb.

[30] J. Wamburu, S. Lee, P. Shenoy, D. Irwin, Analyzing distribution transformers at city scale and the impact of EVs and storage, in: Proceedings of the Ninth International Conference on Future Energy Systems, ACM, 2018, pp. 157-167.

[31] B. Witkamp, R. van Gijlswijk, M. Bolech, T. Coosemans, N. Hooftman, The Transition to a Zero Emission Vehicles Fleet for Cars in The EU by 2050: Pathways and Impacts: An Evaluation of Forecasts and Backcasting the COP21 Commitments: A Policy Support Study Carried Out as Part of the EAFO Project for The European Commission Directorate General Mobility \& Transport, Tech. Rep., EAFO; European Commission, 2017.

[32] W. Folkerts, W. van Sark, C. de Keizer, W. van Hooff, M. Donker, Roadmap PV Systems and Applications, SEAC, 2017.

[33] M. Van Der Kam, W. van Sark, Smart charging of electric vehicles with photovoltaic power and vehicle-to-grid technology in a microgrid; a case study, Appl. Energy 152 (2015) 20-30.

[34] S.-H. Liao, J.-H. Teng, C.-K. Wen, Developing a smart charger for EVs' charging impact mitigation, in: 2015 IEEE 2nd International Future Energy Electronics Conference, IFEEC, IEEE, 2015, pp. 1-6.

[35] M. Spöttle, K. Jörling, M. Schimmel, M. Staats, L. Grizzel, L. Jerram, W. Drier, J. Gartner, Research for TRAN Committee-Charging Infrastructure for Electric Road Vehicles, European Parliament, 2018. 